\title{
Avaliação da composição macroelementar do osso terceiro metacarpiano de equinos
}

\author{
Evaluation of the macro elemental composition of the equine third metacarpal bone
}

\author{
Mariana Baroni SELIM'; Taís MOTA ; Jim Heiji ABURAYA²; Thales dos Anjos de Faria VECHIATO³; \\ André Luis do Valle De ZOPPA $^{1}$ \\ ${ }^{1}$ Departamento de Cirurgia da Faculdade de Medicina Veterinária e Zootecnia da Universidade de São Paulo, São Paulo - SP, \\ Brasil \\ ${ }^{2}$ Médico Veterinário e Doutor em Física, Faculdade de Medicina Veterinária e Zootecnia/Instituto de Física, Universidade de São \\ Paulo, São Paulo - SP, Brasil \\ ${ }^{3}$ Mestre em Clínica Médica da Faculdade de Medicina Veterinária e Zootecnia da Universidade de São Paulo, São Paulo - SP, \\ Brasil
}

\begin{abstract}
Resumo
O terceiro osso metacarpiano equino é uma região de particular interesse devido ao grande número de lesões a que está sujeito. Assim, justifica-se o estudo detalhado da sua estrutura anatômica, dimensões e composição elementar para o aprimoramento de técnicas de diagnóstico, tratamento e prevenção de lesões. Neste trabalho,foi estudada a composição macroelementar óssea de 30 pares de osso terceiro metacarpiano equino e sua relação com a idade, sexo, peso, raça, alimentação e atividade dos animais. As concentrações médias de cálcio e fósforo das amostras foram $205 \pm 62 \mathrm{mg} / \mathrm{g}$ e $97 \pm 32 \mathrm{mg} / \mathrm{g}$, respectivamente. A relação [Ca]/[P] encontrada foi de 2,12 $\pm 0,13$, indicando que a proporção entre o cálcio e fósforo no tecido ósseo é constante e aproximadamente igual à razão 2:1. A análise estatística dos dados mostrou normalidade para todo o conjunto de medidas. A avaliação entre os animais com atividade física intensa e os animais com atividade física leve, em que a quantidade de material mineralizado para o primeiro e segundo conjuntos são, respectivamente, $[\mathrm{Ca}]=222 \pm 57 \mathrm{mg} / \mathrm{g}$ e $[\mathrm{Ca}]=179 \pm 47 \mathrm{mg} / \mathrm{g}$, evidenciou a existência de correlação estatística entre o depósito de material mineral e a função exercida pelos animais. Para as demais características, não foram verificadas evidências estatísticas de inter-relações. Tais achados serão subsídios para estudos posteriores acerca de possíveis correlações com enfermidades de origem fisiológica ou nutricional frente à casuística de fraturas e outras afecções comuns ao aparelho locomotor equino.
\end{abstract}

Palavras-chave: Equino. Composição. Mineral. Osso. Metacarpiano.

\begin{abstract}
The equine third metacarpal bone has been seen as a region of particular interest due to the large number of injuries to which it is subject. Thus, the detailed study of its anatomical structure, size and elemental composition is justified for the improvement of diagnostic techniques, treatment and prevention of injuries. In this work it has been studied the macro elemental composition of 30 pairs of equine third metacarpal bone and its relation with age, sex, weight, breed, nutrition and activity of animals. The average concentrations of calcium and phosphorus of the samples were $205 \pm 62 \mathrm{mg} / \mathrm{g}$ and $97 \pm 32 \mathrm{mg} / \mathrm{g}$, respectively. The ratio [Ca] / [P] was found to be $2.12 \pm 0.13$, indicating that the ratio of calcium and phosphorus in bone tissue is constant and approximately equal to the ratio $2: 1$. The statistical analysis showed normal values for the entire set of measurements. In the evaluation of the animals with intense physical activity, and animals with light physical activity, it was found that the amount of mineralized material for the first and second sets respectively $([\mathrm{Ca}]=222 \pm 57 \mathrm{mg} / \mathrm{g}[\mathrm{Ca}]=179 \pm 47 \mathrm{mg} / \mathrm{g})$, showing the existence of statistical correlation between the deposit of mineral material and the function performed by the animals. For the other characteristics were not found statistical evidence of correlations. These findings will be input for further studies of possible correlations with diseases of nutritional origin or physiological outside the series of fractures and other disease common to the equine locomotor system.
\end{abstract}

Keywords: Equine. Composition. Mineral. Bone. Metacarpus.

Cavalos atletas, particularmente os de corrida, estão sujeitos a grandes cargas de pressão em seus ossos e articulações. O terceiro osso metacarpiano tem sido visto como uma região de particular interesse devido ao grande número de lesões ao qual está sujeito ${ }^{1}$ e pelo
Correspondência para:

Mariana Baroni Selim

Rua Maracá, 132 ap. 61

CEP: 04313-210 - Vila Guarani - São Paulo/SP/Brasil

e-mail: marianabaroniselim@yahoo.com.br

Recebido: 09/09/2011

Aprovado: 27/06/2012 
fato de que há pouco tecido adjacente recobrindo-o e protegendo- $\mathrm{O}^{2}$.

O sucesso na realização de osteossíntese na espécie equina ainda é insatisfatório quando comparado a outras espécies. Uma das maiores dificuldades para a reparação de fraturas em cavalos é a incapacidade de se manter a fixação, pois, muitas vezes, os materiais implantados não são resistentes o suficiente para manter a sua integridade até que a reparação óssea se complete. Este resultado, dentre outros fatores, tem relação direta com o reduzido número de projetos de pesquisa acerca da anatomia, bem como da composição química e das dimensões das estruturas, o que acaba impossibilitando o desenvolvimento de implantes adequados para a reparação óssea em equinos² ${ }^{2}$

As afecções do osso terceiro metacarpiano são motivo de grandes perdas financeiras para criadores e atletas do meio hípico, seja pela perda da função do animal, ou pelas despesas com tratamento e reabilitação.

O conhecimento das variáveis anteriormente citadas, e também da capacidade óssea de resistir aos traumas, assim como o aperfeiçoamento da técnica cirúrgica e implantes, possibilitarão resultados mais satisfatórios, diminuindo as perdas econômicas decorrentes das fraturas em equinos.

O presente estudo visou à determinação da composição macroelementar do osso terceiro metacarpiano equino em relação ao sexo, raça, idade, peso, dieta e atividade física dos animais.

Foram coletados 30 pares de osso terceiro metacarpiano equino, provenientes do Serviço de Patologia da Faculdade de Medicina Veterinária e Zootecnia da USP. Na confecção das amostras, seguiu-se as técnicas recomendadas por Falla-Sotello et al. ${ }^{3}$ e Santos et al. .

O processo de preparo das amostras envolveu a dissecação dos ossos, obtenção de fragmentos,remoção da matriz orgânica e a confecção de pastilhas para posterior análise da composição macroelementar (concentrações de cálcio e fósforo).
A análise da composição elementar foi efetuada a partir da técnica PIXE (Particle Induced X-ray Emission) no Laboratório de Análise de Materiais e Feixes Iônicos (LAMFI) do Instituto de Física da Universidade de São Paulo (IFUSP).

Para verificar a existência de correlação entre a composição mineral, a idade e o peso dos animais, foi utilizado o teste de correlação de Pearson. Visando verificar possíveis efeitos do sexo, raça, dieta e atividade física sobre a composição mineral do terceiro metacarpiano, foi utilizado o teste $t$ de Student.

Os animais foram divididos em subgrupos dentro dos grupos de raças, dieta e atividades físicas para possibilitar a aplicação dos testes estatísticos. De acordo com as raças, os animais foram separados em dois grupos distintos: o de animais com raça definida e o de animais sem raça definida. Quanto à dieta, foram divididos em grupo alimentado com volumoso e grupo alimentado com volumoso e concentrado. Levando em conta a atividade física dos animais, eles foram agrupados de acordo com a intensidade desta. Dessa forma, animais de esporte, trabalho e exposição foram classificados como de atividade física intensa; e animais de passeio e reprodutores foram classificados como de atividade física leve (Tabela 1).

A porcentagem média de cinzas encontrada nas amostras foi de $63,36 \%$ (desvio-padrão 0,03 ). As concentrações médias de cálcio e fósforo ósseo equivalem a $201,69 \mathrm{mg} / \mathrm{g}$ (desvio-padrão 55,32) e $95,48 \mathrm{mg} / \mathrm{g}$ (desvio-padrão 27,97), respectivamente.

Os testes de correlação de Pearson entre a composição mineral, a idade e o peso resultaram em valores de $\mathrm{r}$ equivalentes a $0,025(\mathrm{P}=0,896)$ e $0,235(\mathrm{p}=0,211)$, respectivamente. $\mathrm{O}$ teste $\mathrm{t}$ entre os grupos de machos e fêmeas resultou em um intervalo de confiança de $-59,5$ a 40,3 ( $\mathrm{P}=0,688)$, com média de 195,3 mg/g (desvio-padrão 63,6) para as fêmeas e $204,9 \mathrm{mg} / \mathrm{g}$ (desvio-padrão 53,7) para os machos.

Em relação à raça dos animais, observou-se um intervalo de confiança variando de $-20,8$ a 
Tabela 1 - Características dos animais utilizados e concentrações de cálcio e fósforo das amostras - São Paulo - 2007-2008

\begin{tabular}{|c|c|c|c|c|c|c|c|c|}
\hline Animal & Raça & Sexo & Idade (anos) & Peso $(\mathrm{Kg})$ & Dieta & Atividade Física & {$[\mathrm{Ca}](\mathrm{mg} / \mathrm{g})$} & {$[\mathrm{P}](\mathrm{mg} / \mathrm{g})$} \\
\hline 1 & Sem Raça Definida & Feminino & 7 & 343 & Volumoso & Leve & 100,76 & 42,63 \\
\hline 2 & Com Raça Definida & Masculino & 10 & 467 & Volumoso e Concentrado & Intensa & 220,26 & 104,96 \\
\hline 3 & Com Raça Definida & Feminino & 12 & 408 & Volumoso e Concentrado & Intensa & 209,83 & 97,71 \\
\hline 4 & Com Raça Definida & Feminino & 2 & 350 & Volumoso e Concentrado & Leve & 273,75 & 127,97 \\
\hline 5 & Sem Raça Definida & Feminino & 14 & 384 & Volumoso e Concentrado & Leve & 210,58 & 96,17 \\
\hline 6 & Com Raça Definida & Masculino & 17 & 372 & Volumoso e Concentrado & Intensa & 188,07 & 83,73 \\
\hline 7 & Com Raça Definida & Masculino & 19 & 437 & Volumoso e Concentrado & Intensa & 226,11 & 107,01 \\
\hline 8 & Com Raça Definida & Masculino & 12 & 429 & Volumoso e Concentrado & Intensa & 286,07 & 140,50 \\
\hline 9 & Com Raça Definida & Feminino & 18 & 412 & Volumoso e Concentrado & Leve & 158,87 & 77,61 \\
\hline 10 & Sem Raça Definida & Feminino & 7 & 402 & Volumoso e Concentrado & Leve & 222,25 & 108,59 \\
\hline 11 & Com Raça Definida & Masculino & 11 & 380 & Volumoso e Concentrado & Intensa & 246,01 & 119,26 \\
\hline 12 & Com Raça Definida & Masculino & 12 & 462 & Volumoso e Concentrado & Intensa & 177,38 & 89,67 \\
\hline 13 & Com Raça Definida & Masculino & 8 & 458 & Volumoso & Intensa & 265,09 & 137,06 \\
\hline 14 & Com Raça Definida & Feminino & 5 & 372 & Volumoso e Concentrado & Leve & 121,47 & 65,07 \\
\hline 15 & Com Raça Definida & Masculino & 9 & 448 & Volumoso e Concentrado & Leve & 202,97 & 107,43 \\
\hline 16 & Com Raça Definida & Feminino & 2 & 294 & Volumoso & Intensa & 292,25 & 146,09 \\
\hline 17 & Com Raça Definida & Masculino & 8 & 381 & Volumoso e Concentrado & Intensa & 268,91 & 137,45 \\
\hline 18 & Com Raça Definida & Masculino & 13 & 450 & Volumoso e Concentrado & Leve & 156,77 & 71,89 \\
\hline 19 & Com Raça Definida & Masculino & 9 & 435 & Volumoso e Concentrado & Intensa & 264,98 & 118,87 \\
\hline 20 & Com Raça Definida & Masculino & 4 & 384 & Volumoso e Concentrado & Leve & 174,02 & 77,81 \\
\hline 21 & Com Raça Definida & Masculino & 15 & 418 & Volumoso e Concentrado & Intensa & 247,69 & 110,23 \\
\hline 22 & Com Raça Definida & Masculino & 15 & 407 & Volumoso e Concentrado & Leve & 204,11 & 90,22 \\
\hline 23 & Com Raça Definida & Masculino & 9 & 486 & Volumoso e Concentrado & Intensa & 250,13 & 111,58 \\
\hline 24 & Sem Raça Definida & Feminino & 9 & 394 & Volumoso e Concentrado & Leve & 223,87 & 103,11 \\
\hline 25 & Com Raça Definida & Masculino & 5 & 324 & Volumoso e Concentrado & Leve & 136,96 & 65,07 \\
\hline 26 & Com Raça Definida & Masculino & 4 & 432 & Volumoso e Concentrado & Intensa & 176,62 & 70,63 \\
\hline 27 & Sem Raça Definida & Masculino & 7 & 286 & Volumoso & Intensa & 71,13 & 31,37 \\
\hline 28 & Com Raça Definida & Masculino & 14 & 515 & Volumoso e Concentrado & Leve & 176,01 & 82,97 \\
\hline 29 & Com Raça Definida & Masculino & 11 & 492 & Volumoso e Concentrado & Intensa & 158,37 & 74,34 \\
\hline \multirow[t]{3}{*}{30} & Sem Raça Definida & Feminino & 6 & 312 & Volumoso e Concentrado & Leve & 139,36 & 67,53 \\
\hline & & & & & & Média & 201,69 & 95,48 \\
\hline & & & & & & Desvio-Padrão & 55,32 & 27,97 \\
\hline
\end{tabular}

Fonte: HOVET /FMVZ - USP

$121,7(\mathrm{P}=0,134)$ no teste $\mathrm{t}$, com média de $211,8 \mathrm{mg} / \mathrm{g}$ (desvio-padrão 49,9) para os animais com raça definida e 161,3 mg/g (desvio-padrão 66,8) para os sem raça definida. $O$ teste $t$ para composição mineral dentre os dois grupos de dietas revelou um intervalo de confiança de $-158,9$ a 203,6 ( $\mathrm{P}=0,721)$, com média de 204,7 mg/g (desvio-padrão 45,7 ) para o gru- po alimentado com volumoso e concentrado e 182 $\mathrm{mg} / \mathrm{g}$ (desvio-padrão 112) para o grupo alimentado somente com volumoso.

A comparação entre a composição mineral dentre os dois grupos de atividade física revelou um intervalo de confiança de 4,0 a 82,2 ( $\mathrm{P}=0,032)$, com média de $221,8 \mathrm{mg} / \mathrm{g}$ (desvio-padrão 57,2) para o grupo de 
atividade física intensa e $178,7 \mathrm{mg} / \mathrm{g}$ (desvio-padrão 47) para o grupo de atividade física leve.

A porcentagem média de cinzas das amostras se aproxima dos valores descritos na literatura ${ }^{5,6}$. No entanto, foram observados alguns valores que se situam muito abaixo ou acima da média do grupo de amostras.Isto pode ser explicado por variações intrínsecas de cada indivíduo. É possível que alguns animais apresentem teores mais elevados ou reduzidos de matéria mineral óssea em virtude de sua genética, idade, sexo, peso, raça, dieta e intensidade de atividade física desenvolvida ${ }^{7,8}$.

Em relação às concentrações médias de cálcio e fósforo, observa-se que os valores médios encontrados estão próximos aos intervalos descritos por Grace et al. ${ }^{6}$, os quais variam de $164,3 \mathrm{mg} / \mathrm{g}$ a $189,7 \mathrm{mg} / \mathrm{g}$ para o cálcio e de $80,52 \mathrm{mg} / \mathrm{g}$ a $92,88 \mathrm{mg} / \mathrm{g}$ para o fósforo. No referido estudo,foram obtidas amostras de osso terceiro metacarpiano de equinos com idade média de 150 dias. Além disso, em seu trabalho não é especificada a região óssea da qual foram obtidas as amostras. As diferenças entre os valores encontrados no presente trabalho e no dos autores citados pode residir nestes fatores e também na distinção entre a metodologia empregada.

Quanto à relação entre as concentrações de cálcio e fósforo, é possível afirmar que a proporção entre o cálcio e fósforo no tecido ósseo é constante e equivale a aproximadamente $2: 1$, como descrito por diversos autores.

De acordo com os testes de correlação de Pearson entre a composição mineral, a idade e o peso, é possível afirmar que não existe correlação entre o conteúdo mineral ósseo, a idade e o peso dos animais.

Em estudo prévio9 ${ }^{9}$ também não foi observada correlação entre o conteúdo mineral ósseo e a idade dos animais. Com o passar dos anos, ocorre um aumento do conteúdo mineral, o qual é responsável pela maior rigidez dos ossos. No entanto, tais autores observaram que o aumento do conteúdo mineral está relacionado à maior deposição de osso cortical, e não a alterações na composição da matriz mineral óssea ${ }^{10}$.

O teste $\mathrm{t}$ entre a composição mineral óssea e o sexo dos animais revelou a ausência de interferência desta característica sobre a composição óssea. Esta variou da mesma maneira em ambos os grupos (machos e fêmeas). O mesmo pode ser observado nas comparações feitas entre a composição mineral, a raça e a alimentação dos animais. Não foi evidenciado qualquer grau de relação entre a composição mineral e o fato dos animais terem ou não raça definida, da mesma maneira que a alimentação não exerceu qualquer influência sobre a composição óssea no grupo de animais estudado.

Já a comparação entre a composição mineral dentre os dois grupos de atividade física revelou a existência de associação entre a composição mineral óssea e a intensidade de esforço físico requerido para que o animal desempenhe a sua função, como já havia sido observado por Porr et al. ${ }^{11}$.

De acordo com os resultados obtidos, foi possível comprovar parcialmente a hipótese de que a composição óssea varia de acordo com determinadas características dos indivíduos. Verificou-se que a intensidade da atividade física é capaz de influenciar a distribuição dos componentes da matriz óssea. Tais achados são compatíveis com a conhecida capacidade do tecido ósseo se adaptar física e morfologicamente a diferentes cargas de pressão.

Além disso, os resultados obtidos neste estudo poderão contribuir em outros subsequentes, seja naqueles referentes à biomecânica óssea ou ao desenvolvimento de materiais adequados para a osteossíntese na espécie equina.

\section{Agradecimentos}

Aos laboratórios de Feixes Iônicos do Instituto de Física da USP e de Doenças Nutricionais e Metabólicas da Faculdade de Medicina Veterinária e Zootecnia da USP. 


\section{Referências}

1.SELTZER, K. L.; STOVER, S. M.;TAYLOR, K. T.;WILLITS, N. H. The effect of hole diameter on the torsional mechanical properties of the equine third metacarpal bone. Veterinary Surgery, v. 25, n. 5, p. 371-375, 1996.

2. STASHAK, T. S. Claudicação em equinos segundo Adams. 5. ed. São Paulo: Roca, 2006. p. 761-765.

3. FALLA-SOTELO, F.; RIZZUTO, M. A.; TABACNIKS, M. H.; ADDED, N.; BARBOSA, M. D. L.; ABURAYA, J. H. Analysis and discuss of trace elements in teeth of different animal species. In: REUNIÃO DE TRABALHO SOBRE FÍSICA NUCLEAR NO BRASIL, 27., 2004, Santos. Anais... São Paulo: Livraria da Física, 2007. p. 432.

4. SANTOS, P. R.; ADDED, N.; RIZZUTO, M. A.; ABURAYA, J. H.; BARBOSA, M. D. L. Measurement of $\mathrm{Sr} / \mathrm{Ca}$ ratio in bones as a temperature indicator. Brazilian Journal of Physics, v. 36, n. 4(B), p.1388-1390, 2006.

5. BANKS, J. B. Histologia veterinária aplicada. 2. ed. São Paulo: Manole, 1991. p. 137-301.

6. GRACE, N. D.; PEARCE, S. G.; FIRTH, E. C.; FENNESSY, P. F. Content and distribution of macro- and micro-elements in the body of pasture-fed young horses. Australian Veterinary Journal, v. 77, n. 3, p. 172-176, 1999.

7. CARTER, D. R.; SPENGLER, D. M. Biomechanics of fracture. In: SUMNER-SMITH, G. Bone in clinical orthopedic. $2^{\text {nd }}$ ed. Dübendorf: AO Publishing, 2002. p. 305-335.

8. MCDOWELL, L. R. Minerals in animal and human nutrition. Florida: Academic Press, 1992. p. 1-77.

9. EL SHORAFA, W. M.; FEASTER, J. P.; OTT, E. A. Horse metacarpal bone: ash content, cortical area and failure stress interrelationships. Journal of Animal Science, v. 49, n. 4, p. 979-982, 1979.

10.LAWRENCE, L. A.; OTT, E. A.; MILLER, G. J.; POULOS, P. W.; PIOTROWSKI, G.; ASQUITH, R. L. The mechanical properties of equine third metacarpals as affected by age. Journal of Animal Science, v. 72, n. 10, p. 2617-2623, 1994.

11.PORR, C. A.; KRONFELD, D. S.; LAWRENCE, L. A.; PLEASANT, R. S; HARRIS, P. A. Deconditioning reduces mineral content of the third metacarpal bone in horses. Journal of Animal Science, v. 76, n. 7, p. 1875-1879, 1998. 